\title{
Intraarterial transplantation of human umbilical cord blood mononuclear cells in hyperacute stroke improves vascular function
}

Lei Huang ${ }^{1}$, Yichu Liư ${ }^{2}$, Jianfei Lu ${ }^{1}$ Bianca Cerqueira ${ }^{1,2}$, Vivek Misra ${ }^{3}$ and Timothy Q. Duong ${ }^{1,4^{*}}$

\begin{abstract}
Background: Human umbilical cord blood (hUCB) cell therapy is a promising treatment for ischemic stroke. The effects of hyperacute stem cell transplantation on cerebrovascular function in ischemic stroke are, however, not well understood. This study evaluated the effects of hyperacute intraarterial transplantation of hUCB mononuclear cells (MNCS) on cerebrovascular function in stroke rats using serial magnetic resonance imaging (MRI).

Methods: HUCB MNCs or vehicle were administered to stroke rats via the internal carotid artery immediately after reperfusion at 60 min following ischemia onset. Lesion volumes were longitudinally evaluated by MRI on days 0,2 , 14, and 28 after stroke, accompanied by behavioral tests. Cerebral blood flow (CBF) and cerebrovascular reactivity were measured by perfusion MRI and $\mathrm{CO}_{2}$ functional MRI (FMRI) at 28 days post-stroke; corresponding vascular morphological changes were also detected by immunohistology in the same animals.

Results: We found that CBF to the stroke-affected region at 28 days was improved (normalized CBF value: $1.41 \pm 0$. 30 versus $0.49 \pm 0.07$ ) by intraarterial transplantation of hUCB MNCs in the hyperacute stroke phase, compared to vehicle control. Cerebrovascular reactivity within the stroke-affected area, measured by CBF fMRI, was also increased $(35.2 \pm 3.5 \%$ versus $12.8 \pm 4.3 \%)$, as well as the corresponding cerebrovascular density. Some engrafted cells appeared with microvascular-like morphology and stained positive for von Willebrand Factor (an endothelial cell marker), suggesting they differentiated into endothelial cells. Some engrafted cells also connected to host endothelial cells, suggesting they interacted with the host vasculature. Compared to the vehicle group, infarct volume at 28 days in the stem cell treated group was significantly smaller $\left(160.9 \pm 15.7\right.$ versus $\left.231.2 \pm 16.0 \mathrm{~mm}^{3}\right)$; behavioral deficits were also markedly reduced by stem cell treatment at day 28 (19.5 $\pm 1.0 \%$ versus $30.7 \pm 4.7 \%$ on the foot fault test; $68.2 \pm 4.6 \%$ versus $86.6 \pm 5.8 \%$ on the cylinder test). More tissue within initial perfusion-diffusion mismatch was rescued in the treatment group.
\end{abstract}

Conclusions: Intraarterial hUCB MNC transplantation during the hyperacute phase of ischemic stroke improved cerebrovascular function and reduced behavioral deficits and infarct volume.

Keywords: Umbilical cord blood cell, Stroke, Cell transplantation, CBF, MRI, Vasoreactivity

\footnotetext{
* Correspondence: tim.duong@stonybrookmedicine.edu

${ }^{1}$ Research Imaging Institute, University of Texas Health Science Center, San Antonio, Texas, USA

${ }^{4}$ Radiology, Stony Brook Medicine, Stony Brook, NY, USA

Full list of author information is available at the end of the article
} 


\section{Background}

Cell therapy is a promising treatment for ischemic stroke. Several preclinical stroke studies have shown beneficial effects following transplantation of stem cells derived from various sources, including embryonic tissue [1], adult bone marrow [2], adipose [3], and umbilical cord blood (UCB) $[4,5]$. Amongst these, UCB cells offer several advantages because they are more readily available and have no associated graft-versus-host reactions [6]. Unlike cells derived from embryonic sources, there are no ethical issues with using cells derived from UCB. UCB cells have been used clinically to treat hematological malignancies for more than two decades with a good safety record [7]. Transplantation of human UCB (hUCB) mononuclear cells (MNCs) and their different subcomponents (i.e., hematopoietic stem cells (HSCs), mesenchymal stem cell (MSCs), and endothelial progenitor cells (EPCs)) have also been shown to be effective in animal models of ischemic stroke $[4,5,8]$.

Most preclinical studies evaluated cell administration $24 \mathrm{~h}$ or later after stroke, and reported outcomes using behavioral assessment and histology, and a few used $\mathrm{T}_{2}$-weighted magnetic resonance imaging (MRI) to measure infarct volume $[4,5,9,10]$. In addition to quantifying lesion volume and the tissue at-risk (i.e., "perfusion-diffusion" mismatch) $[11,12]$ in a longitudinal manner, MRI can also be used to measure cerebral blood flow (CBF) and CBF responses to physiological and functional challenges, providing a noninvasive method to study neuron-vascular coupling and hemodynamic regulation after stroke and during recovery [12]. CBF and cerebrovascular reactivity are known to be perturbed after stroke [12-14], and recovery of CBF and cerebrovascular reactivity plays an important role in functional recovery in ischemic stroke [15]. However, the effects of hUCB MNC transplantation on vascular function in vivo (i.e., CBF, cerebrovascular reactivity, and the underlying vascular changes) are not well understood.

The goal of this study was to evaluate the effects of hyperacute intraarterial transplantation of hUCB MNCs on cerebral vascular function in a rat model of focal cerebral ischemia. We hypothesize that such treatment improves $\mathrm{CBF}$ and cerebrovascular reactivity on the stroke-affected region, with reducing behavioral deficits and infarct volume. The intraarterial delivery method was intended to mimic the clinical condition in which stem cell treatment could be administered following mechanical thrombectomy where a catheter is already in place $[16,17]$. The effects of intraarterial stem cell infusion on the ischemic lesion volume were longitudinally evaluated by MRI on days $0,2,14$, and 28 days following stroke, accompanied with behavioral tests. CBF and cerebrovascular reactivity were measured by perfusion MRI and $\mathrm{CO}_{2}$ fMRI at 28 days post-stroke; corresponding vascular morphological changes were also detected by immunohistology in the same animals.

\section{Methods}

All experiments followed guideline and regulations consistent with the Guide for the Care and Use of Laboratory Animals, Public Health Service Policy on Humane Care and Use of Laboratory Animals, and the Animal Welfare Act and Animal Welfare Regulations. Animal experiments were approved by the Institutional Animal Care and Use Committee of the University of Texas Health Science Center San Antonio. Animals arrived at our facility at least 5 days before experimentation. Rats were housed two per cage prior to stroke and one per cage after stroke in a Tecniplast caging system with autoclaved Sani-chip bedding with 12-h light and 12-h dark cycle. Rats had ad libitum access to irradiated rodent chow from Harlan laboratories and autoclaved water. In addition, gel food (Bio-Serv) was provided in the cage after stroke. Buprenex (0.05 mg/kg) was injected subcutaneously for the first 3 days after surgery.

Sample sizes were calculated via Lamorte's Power calculation [18] (University of Boston) with $\alpha=0.05$. Expected variances and differences between groups were derived from pilot experiments or the reports of others [5]. For infarction evaluation, effect size $=1.78$, six animals per group were needed to achieve $>80 \%$ power. For behavior experiments, effect size $=1.95$, six animals per group were needed to achieve $>80 \%$ power. Experiments and analysis were performed in a blinded manner.

\section{Middle cerebral arterial occlusion model and experiment groups}

Twenty male Sprague Dawley rats (250-320 g; Charles River Laboratories, USA) were subjected to transient (60 min) middle cerebral arterial occlusion (MCAO) via intraluminal vascular occlusion, as previously described $[19,20]$. Rats were anesthetized initially with $3.5 \%$ isoflurane mixed with room air and maintained at $2.0 \%$ isoflurane during surgery, and $1.5 \%$ during MRI. The left common carotid artery and external carotid artery (ECA) were exposed through a midline neck incision. A silicon rubber-coated filament (Doccol Corporation, Sharon, MA, USA) was introduced into the left internal carotid artery (ICA) through the ECA stump to occlude the origin of the MCA. After a 60-min occlusion, reperfusion was achieved by filament withdrawal. The rectal temperature was maintained at $37 \pm 0.5{ }^{\circ} \mathrm{C}$. The heart rate and blood oxygen saturation level were monitored using a MouseOx system (STARR Life Science Corp., Oakmont, PA, USA). All recorded physiological parameters were maintained within normal physiological ranges (arterial $\mathrm{O}_{2}$ saturation 91-98\%, heart rate $350-410 \mathrm{bpm})$. 
Stroke rats were randomly assigned to two groups: (1) phosphate-buffered saline (PBS) as a vehicle control; or (2) hUCB MNCs via intraarterial transplantation after reperfusion. In order to minimize the variability of $\mathrm{MCAO}$, the exclusion criteria were that initial apparent diffusion coefficient (ADC) lesion volume at $30 \mathrm{~min}$ after MCAO was $<100 \mathrm{~mm}^{3}$, indicative of incomplete occlusion. One rat from each group died during the follow-up study; the mortality rate $(1 / 10)$ was equal in the two groups. Three rats from each group were excluded due to small initial ADC lesion. The final sample sizes were six rats for each group. The experimental design is shown in Fig. 1.

\section{Cell preparation and transplantation}

Intracarotid cell transplantation was performed immediately after reperfusion. Cryopreserved hUCB MNCs were purchased from StemCell Technologies (\#70007; Vancouver, Canada) which were separated from the cord blood of a healthy donor by density gradient centrifugation. Cells were rapidly thawed at $37{ }^{\circ} \mathrm{C}$ and passed through a sterilized $70-\mu \mathrm{m}$ filter (Thermo Fisher). The cell count of a single cell suspension and viability was quantified by the trypan-blue dye exclusion method. The volume was adjusted for a total amount of $5 \times 10^{6} \mathrm{hUCB}$ MNCs in $35 \mu \mathrm{l}$ PBS. Immediately following withdrawal of the filament, the ipsilateral ECA stump was cannulated with a PE-5 tube which was connected to a 50- $\mu \mathrm{l}$ microneedle Hamilton syringe filled with cell suspension. The distal end of the PE-5 tube was navigated to the extracranial part of the ICA. The pterygopalatine artery was temporarily ligated. A cell suspension of $35 \mu \mathrm{l}$ was infused over the course of $5 \mathrm{~min}$ into the ICA. Rats in the vehicle group were infused with the same volume of PBS.

\section{MRI}

MRI was performed on a Bruker 7-T BioSpec Scanner with a $40 \mathrm{G} / \mathrm{cm}$ BGA12S gradient insert (Billerica, MA,
USA). A custom-made surface coil (2.3-cm internal diameter) and a neck coil were used for brain imaging and perfusion labeling separately. Rectal temperature was monitored and maintained at $37.0 \pm 0.5{ }^{\circ} \mathrm{C}$ during the MRI scan using a thermostatically controlled water flow system. MRI was acquired at 30 min after MCAO and after reperfusion, and again at 2, 14, and 28 days after MCAO.

\section{$A D C$}

Diffusion-weighted images were acquired using a singleshot, spin-echo, echo-planar imaging (EPI) sequence, with the following parameters: matrix $=96 \times 96$, reconstructed to $128 \times 128$, FOV $=2.56 \times 2.56 \mathrm{~cm}$, seven 1.5 $\mathrm{mm}$ slices, $\mathrm{TR}=3 \mathrm{~s}$, and $\mathrm{TE}=37 \mathrm{~ms}$. Two levels of diffusion sensitization $\left(b=0\right.$ and $\left.1200 \mathrm{~s} / \mathrm{mm}^{2}\right)$, applied along the $\mathrm{x}, \mathrm{y}, \mathrm{z}$ direction separately, were used to calculate the ADC map [19]. Total acquisition time $=3.5 \mathrm{~min}$.

\section{CBF}

The continuous arterial spin-labeling (cASL) technique was performed to measure $\mathrm{CBF}$ as previously described $[19,20]$. cASL employed a 2.7-s square radiofrequency pulse to the labeling coil. A single-shot, gradient-echo, EPI sequence was used with the following parameters: matrix $=96 \times 96$, reconstructed to $128 \times 128$, FOV $=$ $2.56 \times 2.56 \mathrm{~cm}$, seven $1.5-\mathrm{mm}$ slices, $\mathrm{TR}=3 \mathrm{~s}$, flip angle $=90^{\circ}$, and $\mathrm{TE}=14 \mathrm{~ms}$. Pair images with and without tagging were acquired. Total acquisition time $=6 \mathrm{~min}$. For CBF, 60 repetitions were obtained and averaged. To investigate the response to hypercapnic challenge at 28 days after stroke, dynamic CBF was acquired for 2 min during air inhalation, then for 3 min during 5\% $\mathrm{CO}_{2}$ (premixed) inhalation, and subsequently $5 \mathrm{~min}$ of inhalation of air [21].

\section{$T 2$}

T2-weighted image was acquired using fast spin-echo sequence, with $\mathrm{TR}=3 \mathrm{~s}$ and four effective $\mathrm{TE}$ (25, 40, 75,

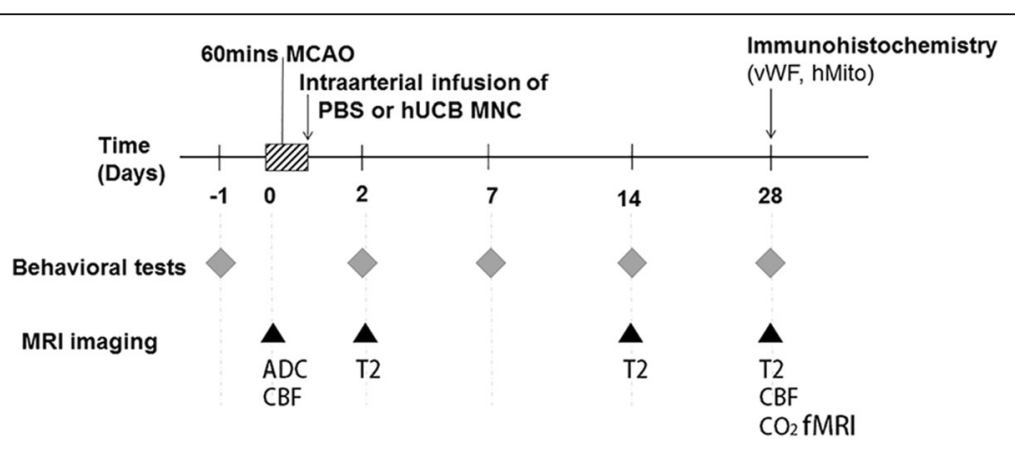

Fig. 1 Timeline of the experiment. Phosphate-buffered saline (PBS) or human umbilical cord blood (hUCB) mononuclear cells (MNCS) were transplanted immediately after 60-min middle cerebral arterial occlusion (MCAO). Behavioral tests and magnetic resonance imaging (MR) were performed at the indicated time points. $A D C$ apparent diffusion coefficient, CBF cerebral blood flow, fMRI functional MRI, hMito human mitochondria, vWF von Willebrand factor 
and $120 \mathrm{~ms}$ ) to generate T2 maps. Other parameters were: seven $1.5-\mathrm{mm}$ coronal images, $\mathrm{FOV}=2.56 \times 2.56 \mathrm{~cm}$, matrix $96 \times 96$, reconstructed to $128 \times 128$, and 8 transients for signal averaging. Total acquisition time $=8 \mathrm{~min}$.

\section{Imaging analysis}

$\mathrm{ADC}, \mathrm{CBF}, \mathrm{T} 2$ map and $\mathrm{CO}_{2}$ reactivity maps were generated and analyzed using Matlab (MathWorks Inc., Natick, MA, USA) and STIMULATE (University of Minnesota) as previously described [19, 22]. Image maps of individual subjects were co-registered across time points by QuickVol and MRIAnalysisPak software. Stroke-induced initial lesion was defined by an abnormal ADC 30 min post-MCAO with an established threshold $\left(0.53 \times 10^{-3} \mathrm{~mm}^{2} / \mathrm{s}\right)$ [23]. The ischemic core and perfusion-diffusion mismatch were defined based on 30-min ADC and CBF maps using previously described measurements $[12,19]$. The fate of the initial ischemic core and mismatch tissues were tracked over time. Lesion volume was calculated based on the T2 map at 2 days post-MCAO due to a better resolution than the ADC map. The lesion area was defined by the pixels with $\mathrm{T} 2$ value higher than the mean value plus two times the standard deviation (mean $+2 \mathrm{SD}$ ) provided by the contralateral side of the brain. Lesion volume was quantified by summing all the lesion areas measured on all slices and multiplying by the slice thickness. Edema correction was performed for lesion calculation of day 2 data as reported previously [19]. The normal CBF value was defined as a range of values two standard deviations above and below the mean (mean $\pm 2 \mathrm{SD}$ ) obtained from the contralateral side. Three regions of interest (ROIs) were generated to separate normal, hyper- and hypoperfusion, based on CBF values. For $\mathrm{CO}_{2}$ reactivity, $\mathrm{CBF}$ percentage change maps were calculated by modeling the time course to the input hypercapnic paradigm using STIMULATE Software, as previously described [22, 24]. Investigators performing image analysis were blinded to the experimental groups.

\section{Histology}

Immunohistology for von Willebrand Factor (vWF) was performed immediately after MRI experiments on day 28 post-MCAO. Briefly, rats were anesthetized and perfused transcardially with $4 \%$ paraformaldehyde (PFA). Brains were removed and postfixed in $4 \%$ PFA for $24 \mathrm{~h}$ at $4{ }^{\circ} \mathrm{C}$ and subsequently cryopreserved in $30 \%$ sucrose for 2 days. Brain samples were frozen in $20-\mu \mathrm{m}$ sections and mounted on gelatin-coated slides. Immunofluorescent staining was performed on a separate section correlating with MRI slices. Briefly, frozen sections were blocked by incubation in 10\% goat serum in PBS after washing with PBS (PH 7.4, containing $0.1 \%$ Tween 20 , $0.3 \%$ Triton X-100). Sections were then incubated with primary antibodies at $4{ }^{\circ} \mathrm{C}$ overnight. Primary antibodies used were rabbit anti-vWF (1:100; Abcam \#ab6994,
Cambridge, MA, USA) and mouse anti-human mitochondria (1:50; Millipore \#MAB1273, Temecula, CA, USA). After washing with PBS, the slides were then incubated with the secondary antibodies goat anti-rabbit Alexa Fluor 488 (1:300) and goat anti-mouse Alexa Fluor 594 (1:300; Invitrogen, USA). Slides were washed and mounted with mounting solution containing 4,6'-diamidino-2-phenylindole (DAPI; Vector Laboratories, H1400). Images were acquired with a Zeiss laser scanning microscope (LSM710) and double-labeling confirmed using z-stacks scan. For each brain sample, two sections with a $0.2-\mathrm{mm}$ gap, correlated to the same level as the MRI image, were used for vWF staining and quantification. On each section, three ROIs (within normal, hyper, and hypoperfusion) were selected based on MRI CBF data. Digital images were captured on four randomly selected fields within each ROI. The mean fluorescent intensity of vWF staining was quantified as previously described [25] using Zen2012. Data from all six samples were averaged and compared between the two groups.

\section{Functional assessment}

The foot-fault test and cylinder test were performed to evaluate the sensorimotor function of the rats at 1 day before surgery and $2,7,14$, and 28 days post-MCAO. The foot-fault test measures the forelimb misplacement on a grid during locomotion. The performance of the rats was videotaped for $5 \mathrm{~min}$ or until 50 steps were taken with one forelimb (non-affected). The total number of steps and number of times each forelimb fell below the grid were counted by an observer blinded to the experimental groups. The percentage of foot-faults for the right forelimb (affected by the stroke) to total steps was calculated and presented as previous reported [26].

The cylinder test were performed to determine the asymmetrical use of the forearm. Animal were video recorded in a transparent cylinder (20-cm diameter by $30-$ $\mathrm{cm}$ height) for $5 \mathrm{~min}$. Forelimb placement on the wall was counted by an observer blinded to the experimental groups. The frequency of left forelimb (unaffected) placement to total placement was calculated and expressed as previous described [26].

\section{Statistical analysis}

A two-way analysis of variance (ANOVA; animal group and different ROIs) with Bonferroni post-hoc test was used to compare behavioral scores, lesion volumes, the percentage of pixels with normal perfusion, hyperperfusion, and hypoperfusion on regions corresponding to $T_{2}$ abnormal maps, $\mathrm{CO}_{2}$-induced $\mathrm{CBF}$ percentage changes in normal perfusion, hyperperfusion, and hypoperfusion regions, and vWF fluorescent density in normal perfusion, hyperperfusion, and hypoperfusion regions between the two animal groups. The unpaired two-tailed $t$ test was 
used to compare percentage of tissue rescued, CBF values, and $\mathrm{CO}_{2}$-induced $\mathrm{CBF}$ percentage changes between the vehicle and treatment group. Values are expressed as mean \pm standard error of the mean (SEM). $P$ values $<0.05$ were taken as statistically significant.

\section{Results}

hUCB MNC transplantation significantly improved functional recovery

The foot-fault scores were not significantly different between the vehicle and treatment group before stroke. They increased on day 2 after stroke, followed by some improvement between 7 to 28 days in both groups (Fig. 2a). Improvement was, however, larger and faster in the treatment compared to the vehicle group. The numbers of foot faults were lower in the treatment compared with the vehicle group on days 7,14 , and 28 post-MCAO $(29.2 \pm 4.3 \%$ versus $48.7 \pm 2.4 \%$ at day $7, P=0.009$ by two-way ANOVA with Bonferroni post-hoc test; $20.0 \pm 2.3 \%$ versus $42.0 \pm 4.0 \%$ at day $14, P=0.006 ; 19.5 \pm 1.0 \%$ versus $30.7 \pm 4.7 \%$ at day $28, P=0.040)$, but not on day $2(P=0.86)$.

The forelimb asymmetry scores were not statistically different between groups before stroke. They increased 2 to 28 days after stroke in both groups (Fig. 2b). The treatment

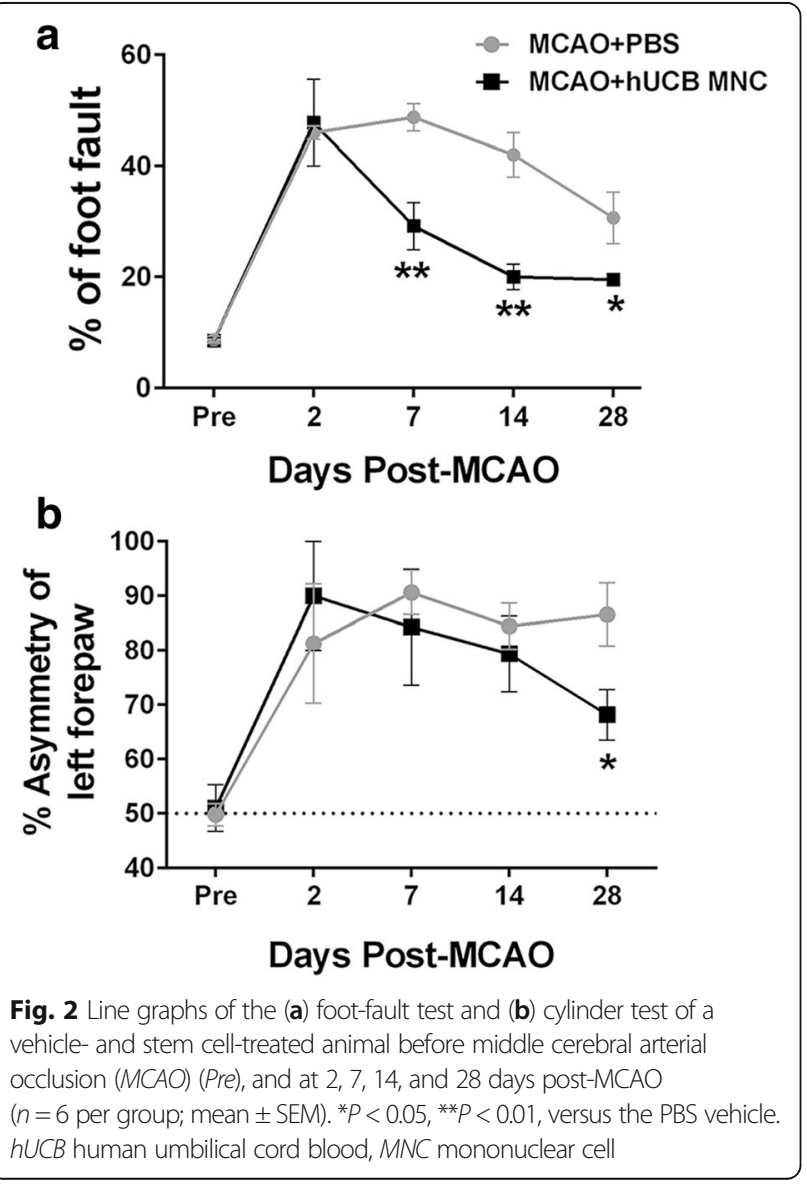

group showed improvement from day 2 to day 28 , reaching significance on day $28(68.2 \pm 4.6 \%$ for treatment group versus $86.6 \pm 5.8 \%$ for PBS-treated group at 28 days, $P=0.04$ by two-way ANOVA with Bonferroni post-hoc test), whereas the vehicle group showed no improvement from day 2 to day 28 . These data show that hyperacute transplantation of hUCB MNCs improved functional recovery at 28 days after stroke.

\section{hUCB MNC transplantation reduced lesion volume}

Ischemic evolution was measured longitudinally by MRI. The initial lesion, determined by $\mathrm{ADC}$ at $30 \mathrm{~min}$ after MCAO, showed no significant difference between groups $(P>0.05$ by two-way ANOVA with Bonferroni post-hoc test; Fig. 3a). By comparison, the $\mathrm{T}_{2}$ infarct volume at 28 days in the treatment group was significantly smaller than that in the vehicle group $(160.9 \pm 15.7$ versus $231.2 \pm 16.0, P=0.04$; Fig. $3 \mathrm{~b})$.

Analysis was performed to evaluate the treatment effects on the initial $(30 \mathrm{~min})$ core and mismatch tissue (Fig. 3c). More initial core and mismatch pixels were rescued in the treatment compared to the vehicle group at 28 days (core: $31.0 \pm 1.4 \%$ versus $26.0 \pm 0.6 \%, P=0.02$; and mismatch: 67.3 $\pm 4.7 \%$ versus $49.0 \pm 2.3 \%, P=0.03$ by unpaired $t$ test). More mismatch tissue was rescued compared to core tissue in the treatment compared to the vehicle group $(P<0.0001)$.

hUCB MNC transplantation increased cerebral blood flow in the infarct area

CBF in abnormal $\mathrm{T}_{2}$ regions (Fig. 4a) was evaluated at 28 days after stroke. Normalized (with respect to the normal hemisphere) CBF values were significantly higher in the treatment compared to the vehicle group $(1.41 \pm 0.30$ versus $0.49 \pm 0.07, \quad P=0.04$ by unpaired $t$ test; Fig. $4 \mathrm{~b}$ ).

We also classified the tissues with different perfusion within the affected area. The treatment group showed more hyperperfusion $(38.8 \pm 10.2 \%$ versus $9.1 \pm 5.0 \%, P=0.02$ by two-way ANOVA with Bonferroni post-hoc test) and less hypoperfusion $(43.1 \pm 3.9 \%$ versus $18.0 \pm 7.5 \%, P=0.029)$ compared to the vehicle group (Fig. 4c).

\section{hUCB MNC transplantation increased cerebral vascular reactivity}

Cerebrovascular reactivity was evaluated using CBF fMRI at 28 days post-MCAO (Fig. 5a). Compared to the contralateral normal hemisphere, $\mathrm{CO}_{2}$-induced $\mathrm{CBF}$ percentage changes were lower in the ipsilateral hemisphere in both groups $(12.8 \pm 4.3 \%$ versus $47.1 \pm 3.1 \%, P=0.001$ by unpaired $t$ test in the vehicle group; $35.2 \pm 3.5 \%$ versus $47.3 \pm 1.0 \%, P=0.012$ in the treatment group). However, CBF percentage changes within the infarcted area were significantly higher in the treatment compared to the vehicle group $(35.2 \pm 3.5 \%$ versus $12.8 \pm 4.3 \%, P=$ 0.016; Fig. 5b). Further analysis showed that $\mathrm{CO}_{2}$-induced 

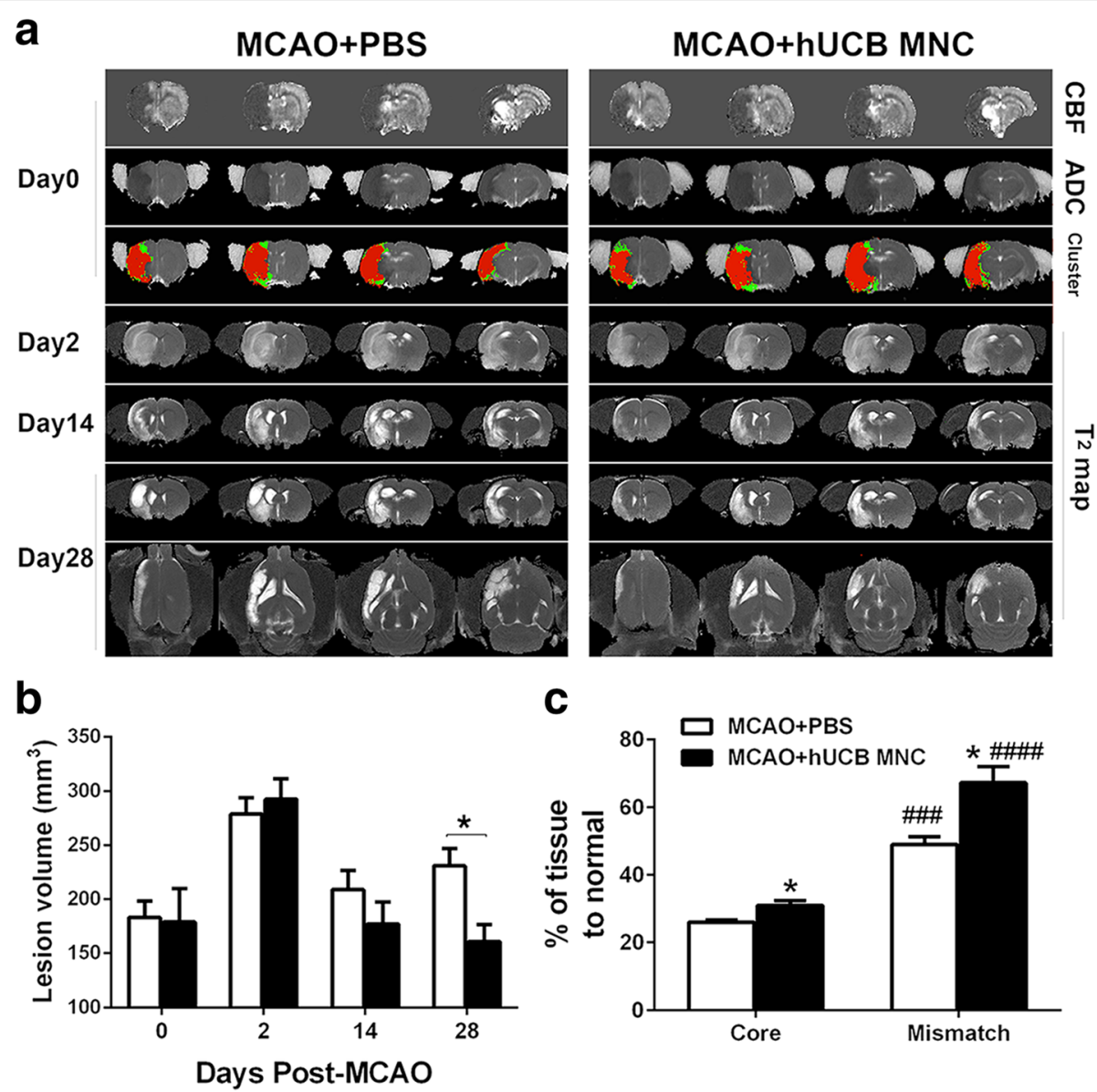

Fig. 3 a Representative cerebral blood flow (CBF), apparent diffusion coefficient ( $A D C$ ), clusters of tissue, and $T_{2}$ map of a PBS vehicle- and stem celltreated animal at day 0 (30 min), and at 2, 14, and 28 days post-middle cerebral arterial occlusion (MCAO). Cluster analysis yielded 'perfusiondiffusion mismatch' (green), and 'ischemic core' (red) clusters, based on initial ADC and CBF data. Hypointense areas on the CBF and ADC map show the initial stroke lesion at day 0 . Hyperintense areas on the $T_{2}$ map indicate stroke lesion at the following time points. $\mathbf{b}$ Quantitative analysis of stroke lesion volume over the time course $\left(n=6\right.$ per group, mean \pm SEM). ${ }^{*} P<0.05$. c Percentage of rescued core and mismatch tissues were analyzed based on day 28 and day 0 MRI data ( $n=6$ per group, mean \pm SEM). ${ }^{*} P<0.05$, versus the PBS vehicle; ${ }^{\# \# \# ~} P<0.001$, $\# \# \# P<0.0001$, versus core tissue of the same group. hUCB human umbilical cord blood, MNC mononuclear cell, PBS phosphate-buffered saline

CBF increases were larger in hyperperfused tissue compared to those in the normal and hypoperfused tissues. Such CBF changes in the hyperperfusion area were more dramatic in the treatment compared to the vehicle group $(48.7 \pm 3.4 \%$ versus $26.6 \pm 5.5 \%, P=0.027$ by two-way ANOVA with Bonferroni post-hoc test; Fig. 5c). $\mathrm{CO}_{2}$-induced CBF percentage changes in the hyperperfusion area were higher than those in the normal perfusion area in the treatment group ( $48.7 \pm 3.4 \%$ versus $25.0 \pm 4.6 \%, P=0.009$ ).

\section{hUCB MNC treatment promoted vascular remodeling after stroke}

To explore possible mechanisms of regional $\mathrm{CBF}$ and vascular reactivity improvements by hUCB MNC treatment, vascular morphology and density of tissues with different CBF was analyzed using vWF immunofluorescent staining (Fig. 6a). vWF staining showed a more defined vascular morphological shape in the treatment group. The mean intensity of vWF fluorescence was significantly higher in the tissue with normal perfusion and hyperperfusion in the treatment group compared to the vehicle group (normal perfusion: $10.9 \pm 0.7$ versus $7.4 \pm 1.0, P=0.031$; hyperperfusion: $15.5 \pm 1.6$ versus $10.6 \pm 0.8, P=0.034$ by two-way ANOVA with Bonferroni post-hoc test; Fig. 6b). Moreover, intensity of vWF from the hyperperfusion area was higher than intensity from the normal perfusion area in the treatment group ( $15.5 \pm 1.6$ versus $10.9 \pm 0.7, P=0.008$ ). These findings suggest hUCB MNC transplantation enhanced vascular density in the infarcted area during stroke recovery.

Engrafted hUCB MNC participated in vasculogenesis after transplantation

To evaluate whether transplanted cells participate in vasculogenesis, double-immunolabeling of the human cell 
a
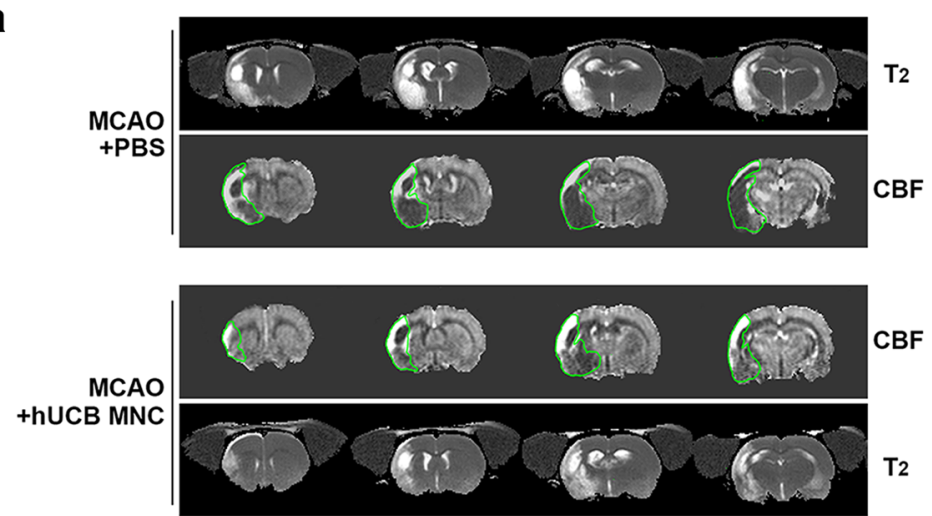

b

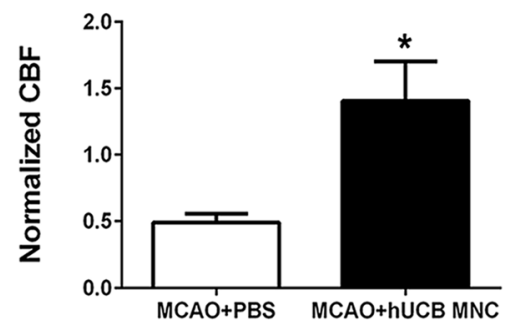

C

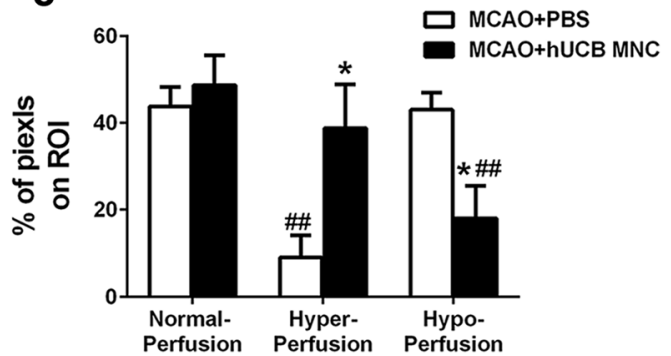

Fig. 4 a Representative cerebral blood flow (CBF) map and corresponding $T_{2}$ map at 28 days post-middle cerebral arterial occlusion (MCAO). The region encircled by the green line is the region of interest $(R O /)$, defined as a $T_{2}$ abnormal area on the corresponding $T_{2}$ map. $\mathbf{b}$ The $C B F$ value of $\mathrm{ROI}$ was quantified. Values were normalized to a homologous region in the contralesional brain. $\mathbf{c}$ The percentage of pixels (with normal CBF, hyperperfusion, and hypoperfusion) on ROI was calculated. The thresholds for abnormal perfusion were defined as the mean CBF value of the contralateral hemisphere \pm 2 standard deviations $\left(n=6\right.$ per group, mean \pm SEM). ${ }^{*} P<0.05$, versus the PBS vehicle;

${ }^{\#} P<0.01$, versus normal perfusion of the same group. $h \cup C B$ human umbilical cord blood, MNC mononuclear cell

\section{a}

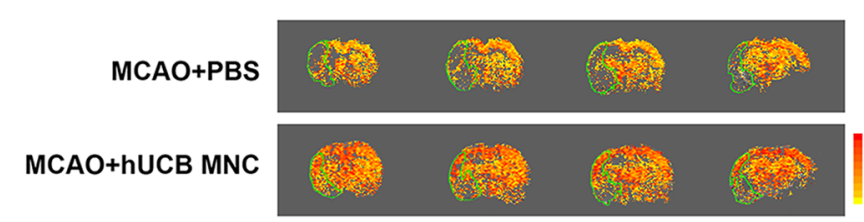

b

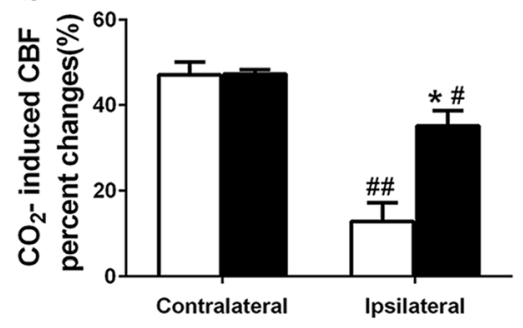

C

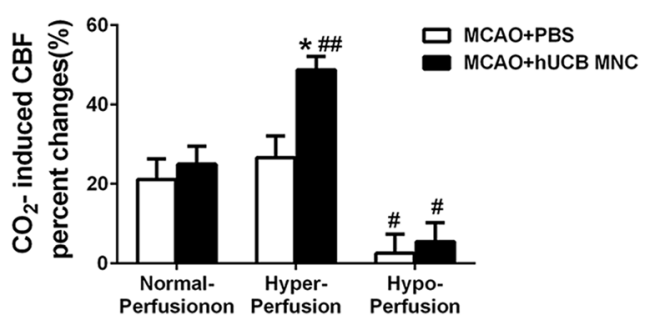

Fig. 5 a Representative $\mathrm{CO}_{2}$ reactivity map from each group at 28 days post-middle cerebral arterial occlusion (MCAO). The region encircled by the green line is the $\mathrm{ROI}$, defined by the stroke lesion on the corresponding $\mathrm{T}_{2}$ map. The color scale bar indicates percentage change in cerebral blood flow $\left(\mathrm{CBF}\right.$ ) ranging from $1 \%$ to $100 \%$ (yellow-red). b Quantification of percentage CBF changes responding to $5 \% \mathrm{CO}_{2}$ challenge from $\mathrm{ROI}$ ( $n=6$ per group, mean \pm SEM). ${ }^{*} P<0.05$, versus the PBS vehicle; ${ }^{\#} P<0.05$, ${ }^{\# \#} P<0.01$, versus contralateral homologous regions. $\mathbf{c} \mathrm{CO}_{2}$ induced percentage change in $\mathrm{CBF}$ were analyzed separately on normal, hyper-, and hypoperfusion areas of $\mathrm{ROI}(n=6$ per group, mean $\pm \mathrm{SEM}) .{ }^{*} P<0.05$, versus the PBS vehicle; ${ }^{\#} P<0.05$, ${ }^{\# \# P}<0.01$, versus normal perfusion of the same group. $h$ UCB human umbilical cord blood, MNC mononuclear cell 


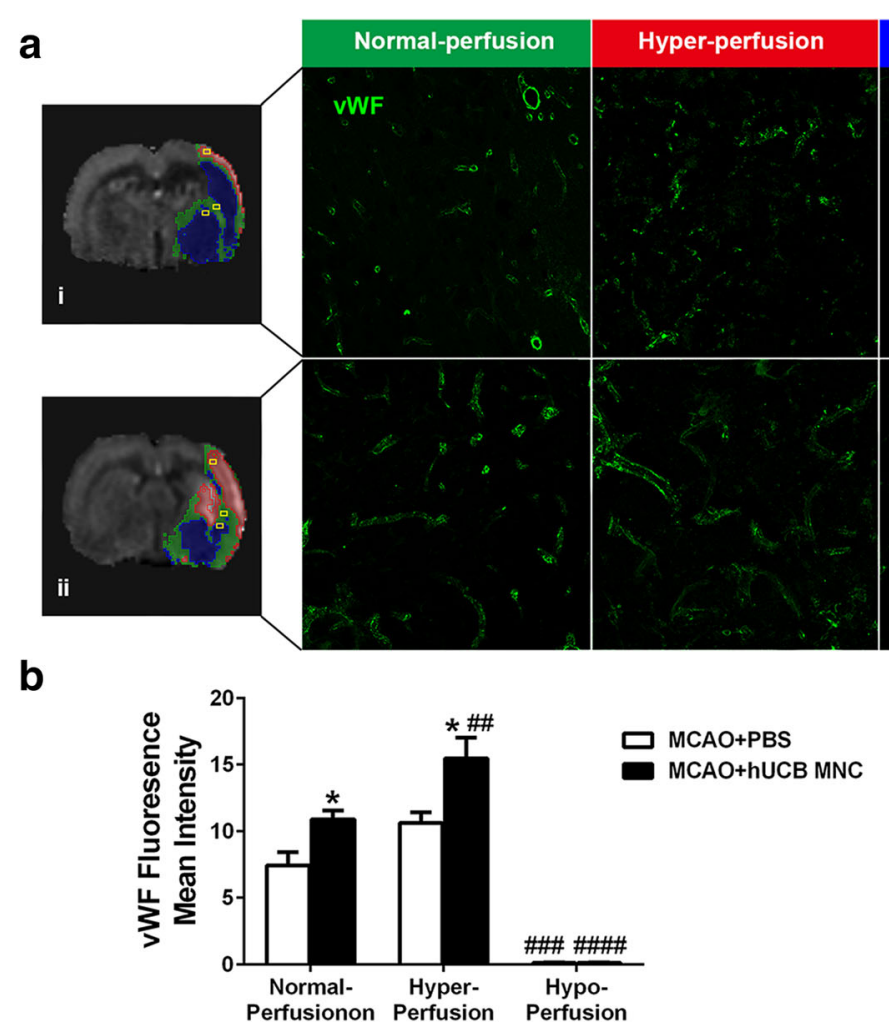

Fig. 6 Vascular remodeling was evaluated by von Willebrand factor (VWF) immunostaining. a Representative perfusion MRI map showing normal, hyper-, and hypoperfusion areas in (i) the vehicle group and (ii) the treatment group at 28 days after stroke. The yellow frame presents the field of view selected in the analysis of VWF immunostaining. Higher magnification images of VWF staining are shown in correlated normal CBF, hyperperfusion, and hypoperfusions areas of the two groups. Scale bar $=100 \mu \mathrm{m}$. b Mean intensity of vWF fluorescence in ROI were quantified $(n=6$, mean \pm SEM).

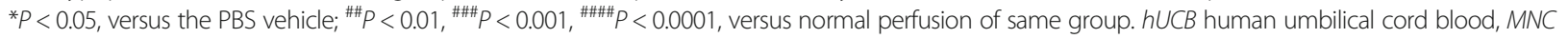
mononuclear cell

marker human mitochondrai (hMito) and vWF were used (Fig. 7). hMito-positive engrafted hUCB MNCs were detected in the ipsilesional cortex at 28 days after transplantation. The hMito-positive engrafted hUCB MNCs appeared as microvascular-like structures and stained positive for vWF, indicating engrafted cells had differentiated into endothelial cells. In addition, some engrafted cells made connections with host endothelial cells (the latter were vWF-positive but hMito-negative), indicating engrafted cells interacted with the host vasculature.

\section{Discussion}

This study provides evidence that intraarterial transplantation of human umbilical cord blood mononuclear cells in the hyperacute phase of ischemic stroke improves regional $\mathrm{CBF}$, cerebrovascular reactivity, and vascular remodeling in which transplanted cells were found to participate in vasculogenesis. Hyperacute stem cell transplantation also reduced behavioral deficits and infarct volume. A novelty of this study is that multimodal MRI was used to track ischemic evolution, verify "patient selection", and evaluate the effects of treatment on different tissue types associated with stem cell transplantation, with corroboration by immunohistology and behavioral function. This study is the first to report the effect of hyperacute intraarterial transplantation of human UCB cells on CBF and vasoreactivity in vivo.

Human umbilical cord blood mononuclear cells (hUCB MNCs) comprise multiple stem/progenitor cells, including HSCs, MSCs, EPCs, and so forth. Many preclinical studies have shown the efficacy of hUCB MNCs for treating stroke, either as hUCB MNCs or separate cell types (e.g., MSCs, EPCs). For example, Boltze et. al. [9] showed that intravenous infusion of hUCB MNCs less than $72 \mathrm{~h}$ after stroke improved recovery of behavioral function. Also, intravenous injection of AC133+ EPCs derived from hUCB cells reduced the infarct volume of stroke rats [27], while a recent study shows that the therapeutic effect of hUCB MNC intraarterial transplantation is better than hUCB-derived MSCs alone [5].

\section{Effects on lesion volume, mismatch evolution, and behavioral function}

Selection of intraarterial rather than intravenous injection was based on the evidence that more engrafted cells 

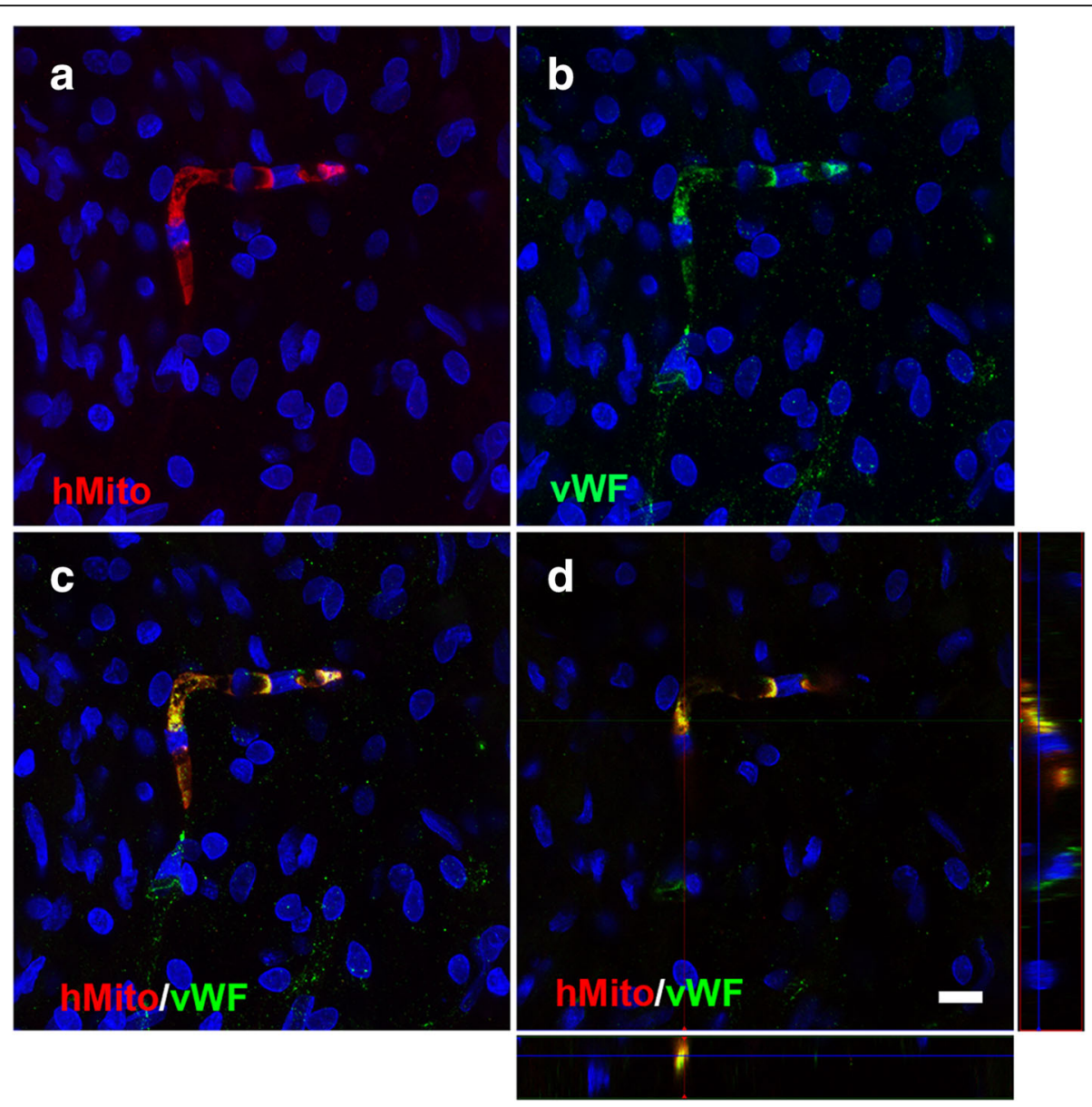

Fig. 7 a Human mitochondrial (hMito) immunofluorescent staining. b The vascular endothelial marker von Willebrand factor ( $v W F)$ immunofluorescent staining. $\mathbf{c}$ Double staining and (d) orthographic projection show co-localization of hMito with vWF on brain slices at 28 days post-MCAO. DAPI (blue) staining was used to identify nuclei. Scale bar $=10 \mu \mathrm{m}$

reach the stroke lesion when transplanted intraarterially [28]. We opted for a dose of $5 \times 10^{6}$ cells for intraarterial injection based on a dose-dependent study that shows intravenous injection of over $10^{6}$ cells 1 day after stroke was sufficient to improve behavioral and histopathologic recovery for cord blood cell transplantation [10]. However, further study is needed to optimize the dosage for intraarterial transplantation in the hyperacute phase of ischemic stroke.

We found hyperacute intraarterial transplantation of hUCB MNCs salvaged more initial diffusion-perfusion mismatch and ischemic core tissue, and reduced infarct volume 28 days after stroke. This approach provides information about the tissue types that are affected by the treatment in a longitudinal fashion, which offers an advantage over terminal histological approaches to evaluate infarct volumes. Also, we used MRI to strictly select 'patients' in this study, which could reduce the variability of experiments and minimize the usage of animals. The improvement in infarct volumes is corroborated by improvement in behavioral scores in the treatment group at 7,14 , and 28 days after stroke. While both foot fault and forelimb asymmetry scores showed improvement in the treatment compared to the vehicle group, there were differences in temporal patterns. For example, in the vehicle group, the foot fault scores improved with time but the forelimb asymmetry scores did not. The difference between groups was smaller in the forelimb asymmetry score on days 7 to 14 . A likely explanation is that the forelimb placement task is less challenging than the foot-fault task, allowing the animals to readily compensate for the deficits; thus, smaller group differences were observed in the forelimb placement task. The same improving trend as for the foot-fault test was found for the cylinder test at 7 and 14 days, even though there was no significant differences between the two groups. This is why multiple behavioral tests were included in the study; the sensitivity of tests differ over time. Reduced behavioral deficits by intraarterial transplantation of hUCB MNCs $24 \mathrm{~h}$ after stroke have been reported up 
to 14 days after stroke [5]. Surprisingly, there was no significant difference on the infarct volume between the two groups at the early time points (day 2 ) after stroke. However, a similar result was reported by Zhu et al. [29] where hUCB MSCs were delivered via intracerebral injection $24 \mathrm{~h}$ after MCAO. The transplantation did not significantly reduce infarct volume at 3 days post-stroke, but it improved functional recovery in mice 14 days post-stroke [29]. This finding likely reflects the therapeutic effect of acute hUCB MNC treatment on improving brain recovery rather than neuroprotection at the acute phase. Based on this, and the nature of cord blood cells, we next focused on the impact of cell transplantation on the cerebrovascular function at the chronic phase of stroke.

\section{Effects on cerebral vasculature}

We found hUCB MNC transplantation increased CBF to the stroke-affected region. Improved perfusion after stroke has been associated with improved functional recovery [30], and this may partly explain the reason for functional improvement in our stem cell-treated group. Furthermore, normalization of CBF improves the delivery of nutrients and oxygen to support brain tissue in the injured area, minimizing neuronal cell death and promoting brain recovery $[31,32]$. Similarly, a tendency for $\mathrm{CBF}$ increase to the stroke-affected hemisphere has been observed at 14 days post-stroke following intravenous injection of hUCB-derived AC133+ EPCs $24 \mathrm{~h}$ after MCAO, even though this trend did not reach significance [27]. Meanwhile, intracerebral transplantation of hUC-derived MSCs has been shown to increase local $\mathrm{CBF}$ in the ischemic hemisphere in stroke rats as measured by laser Doppler flowmetry [33]. MRI as used in our study, in contrast to laser Doppler flowmetry, enables CBF measurements beyond the cortical surface and correlation with perfusion-diffusion mismatch. Furthermore, our results indicate that less tissue suffered hypoperfusion and more tissues experienced hyperperfusion in the stem cell-treated group. A previous study has also demonstrated increased regional hyperperfusion during stroke recovery that may be related to angiogenesis [34].

We also found hUCB MNC transplantation significantly improved cerebral vasoreactivity in the stroke-affected hemisphere. Cerebrovascular reactivity has been showed to be attenuated for an extended period following ischemic injury [35], and improvement in cerebrovascular reactivity has been associated with recovery after stroke $[14,15,36]$. Our findings demonstrate that hUCB MNC treatment has beneficial effects on vascular function in general and likely contributes to stroke recovery and functional improvement at 28 days. It is worth noting that vascular function has been shown to be correlated with stroke recovery [15, 37], and functional MRI is a unique and powerful tool to longitudinally monitor cerebral vascular function in vivo [38]. To our knowledge, this is the first report that early intraarterial transplantation of hUCB MNCs in vivo improves cerebrovascular reactivity in chronic stroke.

To corroborate MRI findings of basal CBF and vasoreactivity improvement, we performed histological experiments in which different ischemic tissue types were chosen for histology based on multimodal MRI. We found more intact vascular morphology and increased vascular density in tissue with hyperperfusion and normal perfusion in the treatment group compared to the vehicle group, suggesting enhanced angiogenesis and vascular remodeling. These findings are consistent with previous reports of enhanced angiogenesis and vascular remodeling after intravenous or intracortical transplantation of UCB cells $[33,39,40]$. The vascular density in tissues with hyperperfusion was higher than that in tissues with normal perfusion, suggesting increased vascular density is correlated with hyperperfusion in subchronic stroke [34, 41].

This improvement is at least in part due to the engrafted hUCB MNCs forming new vessels during stroke recovery, as indicated by the engrafted cells showing microvascular-like structure and expressing the endothelial marker vWF. Moreover, we also found some engrafted cells made connections with host endothelial cells. hUCB cells have been shown to differentiate into endothelial cells and survive for an extended period after transplantation [4, 33, 42]. In addition, engrafted cells could also secrete growth factors, such as angiogenic factors (e.g., vascular endothelial growth factor [43]), which could further contribute to enhanced angiogenesis. Although further mechanistic studies are needed, these findings together suggest hUCB MNCs transplanted early after stroke participate in improving vascular function during recovery.

\section{Limitations of the studies and future directions}

We chose 60-min MCAO as this duration yielded a reasonable volume of initial ischemic lesion and diffusionperfusion mismatch under our experimental conditions [19, 23]. However, different durations of occlusion will need to be explored to mimic various clinical conditions of ischemic stroke. This study used male, healthy young adult rats. Future studies will need to use older animals of both genders and with comorbidities. While we showed that intraarterial hUCB MNC delivery is effective, studies are need to investigate whether hyperacute intravenous cell delivery has a similar effect on vascular function. We chose cell infusion immediately after reperfusion to test the therapeutic potential of combination treatment (thrombectomy and stem cell infusion). Further work is needed to explore the optimized time 
window of HUCB MNC intra-arterial infusion. Although endothelial cell staining using its specific marker (e.g., vWF, CD31) is the widely accepted and used method to study vascular density [25, 27], intravenous perfusion with fluorescent-labeled lectin could show better spatial resolution and connection of the vascular network.

Mechanical thrombectomy is becoming a promising treatment for stroke patients with intracranial large artery occlusions presenting within $6 \mathrm{~h}$ of symptom onset [16]. The time from stroke onset to arterial recanalization significantly impacts outcomes [44], prompting discussion on reorganizing the stroke system of care to facilitate rapid reperfusion and improve prompt access of these therapies to patients in need [45]. Intraarterial infusion of UCB MNCs immediately after mechanical thrombectomy could be a potential adjuvant therapy. The demonstrated improvement in cerebrovascular function is particularly relevant for hyperacute treatment. The allogeneic nature of UCB MNCs could be a practical option for use as an 'off-the-shelf' product readily available for therapeutic use.

\section{Conclusions}

Intraarterial human UCB MNC infusion administered in the hyperacute stroke phase improves regional CBF, cerebrovascular reactivity, and vascular function, while reducing behavioral deficits and infarct volume. The engrafted UCB MNCs differentiate into endothelial cells and interact with the host vasculature. Hyperacute intraarterial infusion of UCB MNCs could serve as a potential adjuvant therapy for post-mechanical thrombectomy.

\section{Abbreviations \\ ADC: Apparent diffusion coefficient; CASL: Continuous arterial spin labeling; CBF: Cerebral blood flow; DAPI: 4',6'-Diamidino-2-phenylindole; ECA: External carotid artery; EPC: Endothelial progenitor cell; fMRI: Functional magnetic resonance imaging; hMito: Human mitochondria; HSC: Hematopoietic stem cell; hUCB: Human umbilical cord blood; ICA: Internal carotid artery; MCAO: Middle cerebral arterial occlusion; MNC: Mononuclear cell; MRI: Magnetic resonance imaging; MSC: Mesenchymal stem cell; PBS: Phosphate-buffered saline; PFA: Paraformaldehyde; ROI: Region of interest; UCB: Umbilical cord blood; vWF: von Willebrand factor}

\section{Acknowledgements}

Not applicable.

\section{Funding}

This work was supported in part by NIH/NINDS (R01-NS45879).

\section{Availability of data and materials}

All data generated or analyzed during this study are included in this published article.

\section{Authors' contributions}

LH: conception and design, collection and/or assembly of data, data analysis and interpretation, manuscript writing, and final approval of manuscript. YL: collection and/or assembly of data, data analysis and interpretation, and final approval of manuscript. JL: collection and/or assembly of data, and final approval of manuscript. BC: data analysis and interpretation, and final approval of manuscript. VM: conception and design, manuscript writing, and final approval of manuscript. TQD: conception and design, financial support, provision of study material, data analysis and interpretation, manuscript writing, and final approval of manuscript. All authors read and approved the final version of the manuscript.

\section{Competing interests}

The authors declare that they have no competing interests.

\section{Consent for publication}

Not applicable.

\section{Ethics approval}

All experiments followed guidelines and regulations consistent with the Guide for the Care and Use of Laboratory Animals, Public Health Service Policy on Humane Care and Use of Laboratory Animals, and the Animal Welfare Act and Animal Welfare Regulations. Animal experiments were approved by the Institutional Animal Care and Use Committee of the University of Texas Health Science Center San Antonio.

\section{Publisher's Note}

Springer Nature remains neutral with regard to jurisdictional claims in published maps and institutional affiliations.

\section{Author details}

${ }^{1}$ Research Imaging Institute, University of Texas Health Science Center, San Antonio, Texas, USA. ${ }^{2}$ Department of Biomedical Engineering, University of Texas, San Antonio, Texas, USA. ${ }^{3}$ Department of Neurology, University of Texas Health Science Center, San Antonio, Texas, USA. ${ }^{4}$ Radiology, Stony Brook Medicine, Stony Brook, NY, USA.

Received: 13 September 2016 Revised: 18 January 2017

Accepted: 4 March 2017 Published online: 22 March 2017

\section{References}

1. Drury-Stewart D, Song M, Mohamad O, Guo Y, Gu X, Chen D, et al. Highly efficient differentiation of neural precursors from human embryonic stem cells and benefits of transplantation after ischemic stroke in mice. Stem Cell Res Ther. 2013;4:93.

2. Chen J, Li Y, Wang L, Lu M, Zhang X, Chopp M. Therapeutic benefit of intracerebral transplantation of bone marrow stromal cells after cerebral ischemia in rats. J Neurol Sci. 2001;189:49-57.

3. Chang KA, Lee JH, Suh YH. Therapeutic potential of human adipose-derived stem cells in neurological disorders. J Pharmacol Sci. 2014;126:293-301.

4. Chen J, Sanberg PR, Li Y, Wang L, Lu M, Willing AE, et al. Intravenous administration of human umbilical cord blood reduces behavioral deficits after stroke in rats. Stroke. 2001;32:2682-8.

5. Karlupia N, Manley NC, Prasad K, Schafer R, Steinberg GK. Intraarterial transplantation of human umbilical cord blood mononuclear cells is more efficacious and safer compared with umbilical cord mesenchymal stromal cells in a rodent stroke model. Stem Cell Res Ther. 2014;5:45.

6. Riordan NH, Chan K, Marleau AM, Ichim TE. Cord blood in regenerative medicine: do we need immune suppression? J Transl Med. 2007;5:8.

7. Ballen KK, Gluckman E, Broxmeyer HE. Umbilical cord blood transplantation: the first 25 years and beyond. Blood. 2013;122:491-8.

8. Rosenkranz K, Meier C. Umbilical cord blood cell transplantation after brain ischemia-from recovery of function to cellular mechanisms. Ann Anat. 2011;193:371-9.

9. Boltze J, Schmidt UR, Reich DM, Kranz A, Reymann KG, Strassburger M, et al. Determination of the therapeutic time window for human umbilical cord blood mononuclear cell transplantation following experimental stroke in rats. Cell Transplant. 2012;21:1199-211.

10. Vendrame M, Cassady J, Newcomb J, Butler T, Pennypacker KR, Zigova T, et al. Infusion of human umbilical cord blood cells in a rat model of stroke dose-dependently rescues behavioral deficits and reduces infarct volume. Stroke. 2004;35:2390-5.

11. Shen Q, Ren H, Fisher M, Bouley J, Duong TQ. Dynamic tracking of acute ischemic tissue fates using improved unsupervised ISODATA analysis of high-resolution quantitative perfusion and diffusion data. J Cereb Blood Flow Metab. 2004;24:887-97.

12. Shen $Q$, Ren $H$, Cheng $H$, Fisher M, Duong TQ. Functional, perfusion and diffusion MRI of acute focal ischemic brain injury. J Cereb Blood Flow Metab. 2005;25:1265-79. 
13. Vorstrup S, Paulson OB, Lassen NA. Cerebral blood flow in acute and chronic ischemic stroke using xenon-133 inhalation tomography. Acta Neurol Scand. 1986;74:439-51.

14. Olah $\mathrm{L}$, Franke $\mathrm{C}$, Schwindt $\mathrm{W}$, Hoehn $\mathrm{M}$. $\mathrm{CO}_{2}$ reactivity measured by perfusion MRI during transient focal cerebral ischemia in rats. Stroke. 2000;31:2236-44.

15. Troisi E, Matteis M, Silvestrini M, Paolucci S, Grasso MG, Pasqualetti P, et al. Altered cerebral vasoregulation predicts the outcome of patients with partial anterior circulation stroke. Eur Neurol. 2012;67:200-5.

16. Powers WJ, Derdeyn CP, Biller J, Coffey CS, Hoh BL, Jauch EC, et al. 2015 American Heart Association/American Stroke Association Focused Update of the 2013 Guidelines for the Early Management of Patients With Acute Ischemic Stroke Regarding Endovascular Treatment: a guideline for healthcare professionals from the American Heart Association/American Stroke Association. Stroke. 2015;46:3020-35.

17. Sutherland BA, Neuhaus AA, Couch Y, Balami JS, DeLuca GC, Hadley G, et al. The transient intraluminal filament middle cerebral artery occlusion model as a model of endovascular thrombectomy in stroke. J Cereb Blood Flow Metab. 2016;36:363-9.

18. Dell RB, Holleran S, Ramakrishnan R. Sample size determination. ILAR J. 2002; 43:207-13.

19. Shen Q, Du F, Huang S, Rodriguez P, Watts LT, Duong TQ. Neuroprotective efficacy of methylene blue in ischemic stroke: an MRI study. PLoS One. 2013;8, e79833.

20. Rodriguez P, Jiang Z, Huang S, Shen Q, Duong TQ. Methylene blue treatment delays progression of perfusion-diffusion mismatch to infarct in permanent ischemic stroke. Brain Res. 2014;158:144-9.

21. Huang S, Du F, Shih YY, Shen Q, Gonzalez-Lima F, Duong TQ. Methylene blue potentiates stimulus-evoked fMRI responses and cerebral oxygen consumption during normoxia and hypoxia. Neuroimage. 2013;72:237-42.

22. Long JA, Watts LT, Li W, Shen Q, Muir ER, Huang S, et al. The effects of perturbed cerebral blood flow and cerebrovascular reactivity on structural MRI and behavioral readouts in mild traumatic brain injury. J Cereb Blood Flow Metab. 2015;35:1852-61

23. Meng X, Fisher $M$, Shen Q, Sotak CH, Duong TQ. Characterizing the diffusion/perfusion mismatch in experimental focal cerebral ischemia. Ann Neurol. 2004:55:207-12

24. Chandra SB, Mohan S, Ford BM, Huang L, Janardhanan P, Deo KS, et al. Targeted overexpression of endothelial nitric oxide synthase in endothelial cells improves cerebrovascular reactivity in Ins2Akita-type-1 diabetic mice. J Cereb Blood Flow Metab. 2016;36:1135-42.

25. Yan T, Venkat P, Chopp M, Zacharek A, Ning R, Cui Y, et al. Neurorestorative therapy of stroke in type 2 diabetes mellitus rats treated with human umbilical cord blood cells. Stroke. 2015;46:2599-606.

26. Talley Watts L, Long JA, Chemello J, Van Koughnet S, Fernandez A, Huang S, et al. Methylene blue is neuroprotective against mild traumatic brain injury. J Neurotrauma. 2014;31:1063-71.

27. Iskander A, Knight RA, Zhang ZG, Ewing JR, Shankar A, Varma NR, et al. Intravenous administration of human umbilical cord blood-derived AC133+ endothelial progenitor cells in rat stroke model reduces infarct volume: magnetic resonance imaging and histological findings. Stem Cells Trans Med. 2013:2:703-14.

28. Pendharkar AV, Chua JY, Andres RH, Wang N, Gaeta X, Wang H, et al. Biodistribution of neural stem cells after intravascular therapy for hypoxic-ischemia. Stroke. 2010;41:2064-70.

29. Zhu J, Liu Q, Jiang Y, Wu L, Xu G, Liu X. Enhanced angiogenesis promoted by human umbilical mesenchymal stem cell transplantation in stroked mouse is Notch1 signaling associated. Neuroscience. 2015;290:288-99.

30. Fujita $Y$, Ihara M, Ushiki T, Hirai H, Kizaka-Kondoh S, Hiraoka M, et al. Early protective effect of bone marrow mononuclear cells against ischemic white matter damage through augmentation of cerebral blood flow. Stroke. 2010;41:2938-43.

31. Rosenthal RE, Silbergleit R, Hof PR, Haywood Y, Fiskum G. Hyperbaric oxygen reduces neuronal death and improves neurological outcome after canine cardiac arrest. Stroke. 2003:34:1311-6.

32. Shin HK, Dunn AK, Jones PB, Boas DA, Lo EH, Moskowitz MA, et al. Normobaric hyperoxia improves cerebral blood flow and oxygenation, and inhibits peri-infarct depolarizations in experimental focal ischaemia. Brain. 2007:130:1631-42
33. Ding DC, Shyu WC, Chiang MF, Lin SZ, Chang YC, Wang HJ, et al. Enhancement of neuroplasticity through upregulation of beta1-integrin in human umbilical cord-derived stromal cell implanted stroke model. Neurobiol Dis. 2007:27:339-53.

34. Lin TN, Sun SW, Cheung WM, Li F, Chang C. Dynamic changes in cerebral blood flow and angiogenesis after transient focal cerebral ischemia in rats. Evaluation with serial magnetic resonance imaging. Stroke. 2002;33:2985-91.

35. Schmidt-Kastner R, Grosse Ophoff B, Hossman KA. Delayed recovery of CO2 reactivity after one hour's complete ischaemia of cat brain. J Neurol. 1986;233:367-9.

36. Suh JY, Shim WH, Cho G, Fan X, Kwon SJ, Kim JK, et al. Reduced microvascular volume and hemispherically deficient vasoreactivity to hypercapnia in acute ischemia: MRI study using permanent middle cerebral artery occlusion rat model. J Cereb Blood Flow Metab. 2015;35:1033-43.

37. Prakash R, Li W, Qu Z, Johnson MA, Fagan SC, Ergul A. Vascularization pattern after ischemic stroke is different in control versus diabetic rats: relevance to stroke recovery. Stroke. 2013;44:2875-82.

38. Lu H, Liu P, Yezhuvath U, Cheng Y, Marshall O, Ge Y. MRI mapping of cerebrovascular reactivity via gas inhalation challenges. J Vis Exp. 2014;(94). doi:10.3791/52306.

39. Lin YC, Ko TL, Shih YH, Lin MY, Fu TW, Hsiao HS, et al. Human umbilical mesenchymal stem cells promote recovery after ischemic stroke. Stroke. 2011:42:2045-53.

40. Taguchi A, Soma T, Tanaka H, Kanda T, Nishimura H, Yoshikawa H, et al, Administration of CD34+ cells after stroke enhances neurogenesis via angiogenesis in a mouse model. J Clin Invest. 2004;114:330-8.

41. Hayward NM, Yanev P, Haapasalo A, Miettinen R, Hiltunen M, Grohn O, et al. Chronic hyperperfusion and angiogenesis follow subacute hypoperfusion in the thalamus of rats with focal cerebral ischemia. J Cereb Blood Flow Metab. 2011;31: 1119-32.

42. Ingram DA, Mead LE, Tanaka H, Meade V, Fenoglio A, Mortell K, et al. Identification of a novel hierarchy of endothelial progenitor cells using human peripheral and umbilical cord blood. Blood. 2004;104:2752-60.

43. Arien-Zakay H, Lecht S, Bercu MM, Tabakman R, Kohen R, Galski H, et al. Neuroprotection by cord blood neural progenitors involves antioxidants, neurotrophic and angiogenic factors. Exp Neurol. 2009;216:83-94.

44. Sheth SA, Jahan R, Gralla J, Pereira VM, Nogueira RG, Levy El, et al. Time to endovascular reperfusion and degree of disability in acute stroke. Ann Neurol. 2015;78:584-93.

45. Fargen KM, Jauch E, Khatri P, Baxter B, Schirmer CM, Turk AS, et al. Needed dialog: regionalization of stroke systems of care along the trauma model. Stroke 2015:46:1719-26

\section{Submit your next manuscript to BioMed Central and we will help you at every step:}

- We accept pre-submission inquiries

- Our selector tool helps you to find the most relevant journal

- We provide round the clock customer support

- Convenient online submission

- Thorough peer review

- Inclusion in PubMed and all major indexing services

- Maximum visibility for your research

Submit your manuscript at www.biomedcentral.com/submit
) Biomed Central 